\title{
A Weight Adaptation Method for Fuzzy Cognitive Maps to a Process Control Problem
}

\author{
Elpiniki Papageorgiou and Peter Groumpos \\ Laboratory for Automation and Robotics \\ Department of Electrical and Computer Engineering \\ University of Patras, Rion 26500, GREECE \\ Tel. +30 2610997293 , Fax. +30 26120997309 \\ \{epapageo, groumpos\} @ee.upatras.gr
}

\begin{abstract}
Fuzzy Cognitive Maps constitute an attractive modeling approach that encompasses advantageous features. The most pronounces are the flexibility in system design, model and control, the comprehensive operation and the abstractive representation of complex systems. The main deficiencies of FCMs are the critical dependence on the expert's opinion and the potential convergence to undesired steady states. In order to overcome these deficiencies a possible solution is the utilization of learning methods to adapt the causeeffect relationships of the FCM model. This research work examines the formulation of a weight adaptation method suitable for FCMs based on nonlinear Hebbian-type learning algorithm. A process control problem is presented and its control is investigated using the adaptation technique.
\end{abstract}

Keywords: Learning algorithms, Nonlinear Hebbian learning, fuzzy logic, fuzzy cognitive maps, process control

\section{Introduction}

Conventional methods were used, successfully, to model and control systems, in the past years, but their contribution is limited in the representation, analysis and solution of complex systems. In such systems, the inspection of their operation depends on human knowledge. Generally, there is a great demand for the developing of autonomous complex systems that can be achieved taking advantage of human like reasoning. Fuzzy Cognitive Maps (FCMs) seem to be capable to deal with situations where the human reasoning process for any procedure includes uncertain descriptions.

FCMs are important modeling means for describing particular domains showing the concepts (variables) and the relationships between them. FCMs can contribute to the construction of more intelligent systems since a more intelligent a system becomes, the more symbolic and fuzzy representations it utilizes.

Over the last 10 years, a variety of FCMs have been used for representing knowledge and artificial intelligence in engineering applications, for instance, fault detection [1], geographical information systems [2] and modelling the supervision of distributed systems [3]. FCMs have been also used for several tasks like web data mining [4], and medical decision-making [5]. 
In this research work we propose a learning methodology for fine-tuning FCM causal links in order to update the initial experts' knowledge and to control a process problem efficiently. In general, experts in a certain domain make the task of creating Fuzzy Cognitive Maps (FCMs), but it is very promising the learning of FCMs from the initial experts' knowledge and experience. Till today, very few research efforts have been made to investigate and propose a learning algorithm suitable for FCMs $[6,7,8,9]$. The introduction of FCM weight adaptation technique eliminates the deficiencies in the usage of FCM and enhances the dynamical behavior and flexibility of the FCM model.

\section{Fuzzy Cognitive Maps Background}

FCMs are special kind of cognitive maps and have been introduced by Kosko [9] to represent fuzzy cause effect relationships instead of the crisp cause-effect relations represented in the original cognitive maps. FCMs are fuzzy signed digraphs with feedback. Nodes in the graph are fuzzy sets and represent concepts. Directed edges represent causal effect relationships between the concepts as the case of generic cognitive maps.

The FCM structure is similar to a recurrent artificial neural network, where concepts are represented by neurons and causal relationships by weighted links connecting the neurons. Concepts reflect characteristics, of the system. Interconnections among concepts of FCM signify the cause and effect relationship that a concept has on the others. These weighted interconnections represent the direction and degree with which concepts influence the value of the interconnected concepts. Figure 1 illustrates the graphical representation of a Fuzzy Cognitive Map. The interconnection strength between two nodes $C_{j}$ and $C_{i}$ is $w_{j i}$, with $w_{j i}$ taking on any value in the range -1 to 1 .

There are three possible types of weights among concepts: $w_{j i}>0$ which indicates positive causality between concepts $C_{j}$ and $C_{i}, w_{j i}<0$ and $w_{j i}=0$.

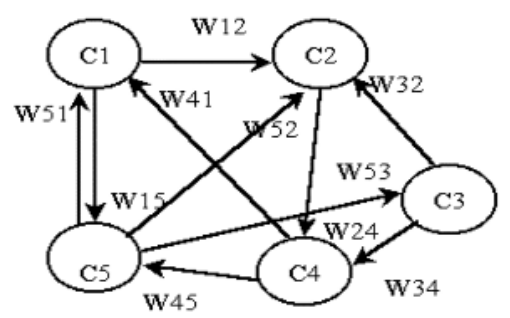

Fig. 1. A simple Fuzzy Cognitive Map

The value of each concept is calculated, computing the influence of other concepts to the specific concept, by applying the following calculation rule:

$$
\mathbf{A}^{(k)}=f\left(\mathbf{A}^{(k-1)}+\sum \mathbf{A}^{(k-1)} \cdot \mathbf{w}\right)
$$


where $\mathbf{A}^{(k)}$ is the vector of values of concepts $C_{i}$ at iteration step k, and $f$ is the sigmoid threshold function $f(x)=1 /\left(1+e^{-x}\right)$.

The output vector $\mathbf{A}$ after the implementation of the threshold function is used as the input vector for the $(\mathrm{k}+1)^{\text {th }}$ stage. This new input vector is again operated on the weight matrix. The following algorithm expresses the FCM interactions.

Algorithm 1: FCM model implications.

Begin

Step 1: Read the input vector $\mathbf{A}^{0}$

Step 2: Give the connection matrix $\mathbf{w}$

Step 3: Calculate the output vector $\mathbf{O}^{(k)}=\mathbf{A}^{(k-1)}+\sum \mathbf{A}^{(k-1)} \cdot \mathbf{w}$

Step 4: Apply threshold to output vector $\mathbf{A}^{(k)}=f\left(\mathbf{O}^{(k)}\right)$

Step 5: If $\left(\mathbf{A}^{(k+1)}=\mathbf{A}^{(k)}\right)$, stop

Else GO TO Step 1.

End

By studying the final state of the iterations, we infer for the model. Equilibrium in the system is attained when we have a set of repeated patterns. Repeated patterns can be fixed points or limit cycles or a chaotic attractor. The "chaotic attractor" is a limit cycle consisting of repeating patterns of differing lengths.

The methodology for developing FCMs is based on a group of experts who are asked to define concepts and describe relationships among concepts and use IF-THEN rules to justify their cause and effect suggestions among concepts and infer a linguistic weight for each interconnection. A more detailed description about the development of FCMs is given in [10].

The most significant weaknesses of the FCMs are their dependence on the expert's opinion and the uncontrollable convergence to undesired states. Learning procedures is a mean to increase the efficiency of FCMs, by modifying the FCM weight matrix.

\section{Learning Methods for Fuzzy Cognitive Maps}

Fuzzy Cognitive Map learning involves updating the strengths of causal links so that FCM converge in a desired region. An advanced learning strategy is to modify FCMs by fine-tuning its initial causal link or edge strengths through training algorithms similar to that in artificial neural networks.

Up-to-date, there have been proposed just a few unsupervised learning algorithms for FCMs $[6,7,8]$ and they are mostly based on ideas coming from the field of artificial neural networks training. Kosko has proposed the first algorithm, named Differential Hebbian Learning, as a form of unsupervised learning, but without any mathematical formulation and implementation in real problems [6]. There is no guarantee that DHL will encode the sequence into the FCM and till today no concrete procedures exist for applying DHL in FCMs. Another method is the Adaptive Random Fuzzy Cognitive Maps (ARFCMs), which are trained with a reinforcement 
learning law. They are based on the theoretical aspects of Random Neural Networks and can be used like associative memories [7]. Despite the recent fusion of FCMs and Random Neural Networks, the resulted Adaptive Random FCM has not any significant results in real dynamic system modeling.

Also, a different approach has been proposed for FCM learning, which is based on evolutionary computation [9]. This technique is exactly the same used in neural networks training. It doesn't take into consideration the initial structure and experts' knowledge for the FCM model and the calculated weights appear large deviations from the actual FCM weights without accepted physical meaning. So, a formal learning methodology suitable for FCMs and for practical applications is still needed.

\section{Weight Adaptation Method Based on Nonlinear Hebbian Learning}

The proposed weight adaptation procedure is based on the Nonlinear Hebbian Learning rule. The proposed nonlinear Hebbian-type rule for ANNs learning [10] have been adapted and modified for the FCM case, determining the Nonlinear Hebbian Learning (NHL) algorithm.

This learning algorithm is based on the premise that all the concepts in FCM model are triggering at each iteration step and change their values. During this triggering process the weight $w_{j i}$ of the causal interconnection of the related concepts is updated and the modified weight $w_{j i}{ }^{(k)}$ is derived for iteration $k$.

The value $A_{i}^{(k+1)}$ of $C_{i}$, concept at iteration $k+1$, is calculated, computing the influence of interconnected concepts with values $A_{j}$ to the specific concept $C_{i}$ due to modified weights $w_{j i}{ }^{(k)}$ at iteration $k$, through the following equation:

$$
A_{i}{ }^{(k+1)}=f\left(A_{i}{ }^{(k)}+\sum_{\substack{j \neq i \\ j=1}}^{N} A_{j}^{(k)} \cdot w_{j i}{ }^{(k)}\right)
$$

Furthermore, some of concepts are defined as Desired Output Concepts (DOCs). These concepts stand for the factors and characteristics of the system that interest us, and we want to estimate their values, which represent the final state of the system. The distinction of FCM concepts as inputs or outputs is determined by the group of experts for each specific problem. experts select the output concepts and they consider the rest as initial stimulators of the system. The learning algorithm that extracts hidden and valuable knowledge of experts can increase the effectiveness of FCMs.

Taking the advantage of the general nonlinear Hebbian-type learning rule for NNs, [10], we introduce the mathematical formalism. The proposed rule has the general mathematical form:

$$
\Delta w_{j i}=\eta_{k} A_{i}\left(A_{j}^{(k)}-w_{j i}{ }^{(k-1)} A_{i}\right)
$$

where the coefficient $\eta$ is a very small positive scalar factor called learning parameter and is determined using experimental trial and error method in order to 
optimize the final solution. We propose the following form of nonlinear weight adaptation algorithm:

$$
w_{j i}{ }^{(k)}=\gamma \cdot w_{j i}{ }^{(k-1)}+\eta^{(k)} A_{i}\left(A_{j}{ }^{(k)}-w_{j i}{ }^{(k-1)} A_{i}\right)
$$

where the $\eta$ is the learning rate parameter and $\gamma$ is the weight decay parameter.

The value of each concept of FCM is updated, through the eq. (2) where the value of weight $w_{j i}{ }^{(k)}$ is calculated using eq. (4). Only the initially non-zero weights suggested by experts are updated for each iteration step. All the other weights remain equal to zero.

Also, we use two criteria functions for the termination of the proposed algorithm. One criterion is the maximization of a function $J$, which has been defined by Hebb's rule in [10]. The $J$ have been proposed for the NHL examining the values of DOCs. The $J$ is defined as: $J=\sum_{i=1}^{l}\left(D O C_{i}\right)^{2}$

where $l$ is the number of DOCs.

The second criterion is the minimization of the variation of two subsequent values of DOCs, represented in equation:

$$
\left|D O C_{j}^{(k+1)}-D O C_{j}^{(k)}\right|<e
$$

in order to terminate the iterative process of the learning algorithm. The term $e$ is a tolerance level keeping the variation of values of DOC(s) as low as possible and it is proposed equal to $e=0.001$, satisfying the termination of iterative process.

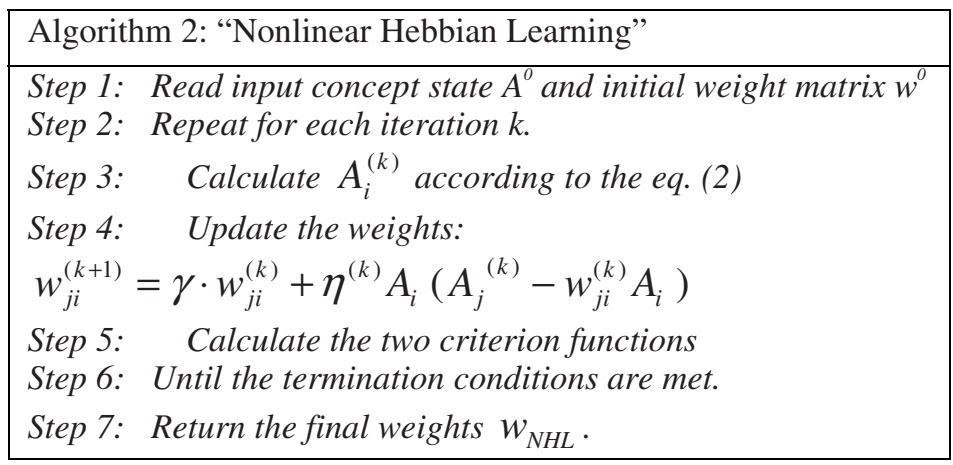

A generic description of the proposed NHL Algorithm for FCMs is given in Algorithm 2. In NHL algorithm, upper and lower bounds for the learning rate parameters $\gamma$ and $\eta$ are determined using trial and error experiments, $0<\eta<0.1$ and $0.9<\gamma<1$, respectively, and then constant values for each one are proposed for each case-study problem.

\section{An Industrial Process Control Problem}

A process control problem encountered in chemical industry is selected to illustrate the workings of the proposed weight adaptation algorithm. The process control 
problem is a part of a chemical plant consisting of two tanks, three valves, one heating element and two thermometers for each tank, as depicted in Figure 2.

For this system a FCM model has been constructed in [3]. Variables and states of the system, such as the height of the liquid in each tank or the temperature, are the concepts of an FCM, which describes the system. For this simple system eight concepts are proposed and they give a good model of the system:

Concept 1: The height of the liquid in tank 1. The height of liquid is dependent on state of valve 1 and valve 2 .

Concept 2: The height of the liquid in tank 2. The height of liquid is dependent on state of valve 2 and valve 3 .

Concept 3: The state of the valve 1 . The valve is open, closed or partially open.

Concept 4: The state of the valve 2. The valve is open, closed or partially open.

Concept 5: The state of the valve 3. The valve is open, closed or partially open.

Concept 6: The temperature of the liquid in tank 1.

Concept 7: The temperature of the liquid in tank 2.

Concept 8: Describes the operation of the heating element, which has different levels of operation and which increases the temperature of the liquid in tank 1.

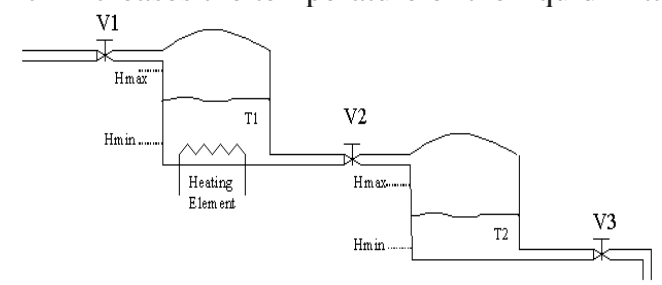

Fig. 2. The illustration for the process control example

These concepts are connected with each other. The signs and the weight of each connection have been determined in [3]. The interconnections among concepts can easily be changed and some new can be added or others can be removed if the human operator decides so, in order to have a better model and operation of the system. Three experts constructed the FCM. The experts determine the cause-effect relationship among concepts. Each connection between concepts has a weight, which ranges between $[-1,1]$ and was determined by a group of experts. Each concept of the FCM takes a value, which ranges in the interval $[0,1]$ and it is obtained after threshold the real measurement of the variable or state, which each concept represent.

The control objective is to keep values of these variables in the following range of values:

$0.5 \leq C 1 \leq 0.75,0.75 \leq C 2 \leq 0.8,0.75 \leq C 6 \leq 0.80,0.65 \leq C 7 \leq 0.70$

The values of the weights will be determined after training the FCM using NHL algorithm. The DOCs in this problem, as experts propose them, are the concepts: $\mathrm{C}_{1}$ of "Tank 1", $\mathrm{C}_{2}$ of "Tank 2", $\mathrm{C}_{6}$ of "Temperature 1" and $\mathrm{C}_{7}$ of "Temperature 2".

Experts have suggested the appropriate initial weights of the FCM model that are shown in the following weight matrix in eq. (8). The desired regions for the DOCs, which reflect the proper operation of the modeled system, have been prespecified in eq. (7). 


$$
\mathbf{w}^{\text {initial }}=\left[\begin{array}{cccccccc}
0 & 0 & 0.21 & 0.38 & 0 & 0 & 0 & 0 \\
0 & 0 & 0 & 0.70 & 0.60 & 0 & 0 & 0 \\
0.76 & 0 & 0 & 0 & 0 & 0 & 0 & 0 \\
-0.80 & 0.70 & 0 & 0 & 0 & 0 & 0.09 & 0 \\
0 & -0.42 & 0 & 0 & 0 & 0 & 0 & 0 \\
0 & 0 & 0.40 & 0 & 0 & 0 & 0 & 0.50 \\
0 & 0 & 0 & 0.30 & 0 & 0 & 0 & 0 \\
0 & 0 & 0 & 0 & 0 & 0 & 0.40 & 0
\end{array}\right]
$$

\section{Simulation Results}

First, before the implementation and testing of the proposed NHL algorithm, we apply the typical Algorithm 1 to find the final state of the iterations in the previously described chemical process control problem. The initial values of concepts given in

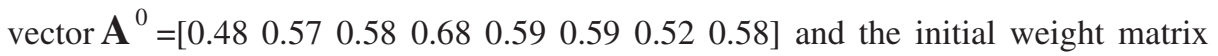
$\mathbf{W}^{\text {initial }}$ are used in Algorithm 1 to calculate the final state of the process. After 10 iterations the equilibrium state is reached $\mathbf{A}^{\text {equil }}=\left[\begin{array}{lll}0.6256 & 0.7330 .76750 .8600 .7704\end{array}\right.$ 0.7390 .6810 .7548 ]. It is observed that the values for $\mathrm{C}_{2}$ and $\mathrm{C}_{6}$, in final state, are out of the suggested desired regions in eq. (7).

Next, the NHL algorithm is applied to train the FCM and modify the weights. This experiment concerns the weight adaptation of the FCM model for the specific case problem to converge in the desired convergence region, determining the appropriate values of cause-effect relationships among concepts (weights). The process starts by applying the same initial vector $\mathbf{A}^{0}$ and weights $\mathbf{w}^{\text {initial }}$. Notably, only the initially non-zero weights that connect the triggering concepts are updated at each iteration step The suggested exponential attenuation value of learning parameter $\eta$ is 0.04 and the associate value for weight decay parameter $\gamma$ is 0.98 , after trial and error experiments, for fast system convergence in desired regions. If the learning parameters $\eta, \gamma$, take different values than the proposed ones, but within the initially suggested limits, then the system will converge in undesired regions.

The proposed algorithmic procedure continues, until the two termination criteria are met. The result of training the FCM is a set of connection weights $w_{j i}$ that satisfies the termination criteria. After 12 iteration cycles, the system converges and the updated weight matrix is the following:

$$
\mathbf{w}_{\text {NHL }}=\left[\begin{array}{cccccccc}
0 & 0 & 0.294 & 0.432 & 0 & 0 & 0 & 0 \\
0 & 0 & 0 & 0.659 & 0.526 & 0 & 0 & 0 \\
0.573 & 0 & 0 & 0 & 0 & 0 & 0 & 0 \\
-0.668 & 0.616 & 0 & 0 & 0 & 0 & 0.217 & 0 \\
0 & -0.171 & 0 & 0 & 0 & 0 & 0 & 0 \\
0 & 0 & 0.441 & 0 & 0 & 0 & 0 & 0.43 \\
0 & 0 & 0 & 0.385 & 0 & 0 & 0 & 0 \\
0 & 0 & 0 & 0 & 0 & 0 & 0.475 & 0
\end{array}\right]
$$


which leads the FCM to the steady state: $C_{1}=0.6197, C_{2}=0.7632, C_{3}=0.7850$, $C_{4}=0.8715, C_{5}=0.7620, C_{6}=0.7512, C_{7}=0.7094, C_{8}=0.7440$

These new values for weights describe new relationships among the concepts of FCM and the DOCs are within the desired regions. Thus the weight adaptation method adjusts the FCM cause-effect relationships and controls the system convergence in accepted steady states.

\section{Conclusions}

In this paper, the unsupervised weight learning method, namely Nonlinear Hebbian Learning, is introduced to adapt the cause-effect relationships between concepts and to eliminate the deficiencies that appear in operation of FCMs. In this way, we take into account the dynamic characteristics of the learning process and the environment.

The proposed method for weight adaptation of FCM is presented and accompanied with the good knowledge of a given system or process can contribute towards the establishment of FCM as a robust technique. Also a formal methodology suitable for process control problems has been developed and desired convergence regions for problem have been produced. In future work, further developments of learning algorithms will be examined.

\section{References}

1. Pelaez C.E. and J.B. Bowles. Using Fuzzy Cognitive Maps as a System Model for Failure Modes and Effects Analysis, Information Sciences, Vol. 88, (1996), 177-199.

2. Liu, Z.Q. \& Satur, R.: Contexual fuzzy cognitive map for decision support in geographical information systems. In: J. of IEEE Trans. On Fuzzy Systems, 7, (1999), 495-507.

3. Stylios, C.D., Groumpos, P.P.: Fuzzy Cognitive Maps in Modeling Supervisory Control Systems, Journal of Intelligent \& Fuzzy Systems, vol. 8, (2000), 83-98.

4. Lee, K.C., Kin, J.S., Chung, N.H. \& Kwon, S.J.: Fuzzy cognitive map approach to webmining inference amplification, In: J. of Expert Systems with Applic., 22, (2002), 197-211.

5. Papageorgiou, E.I., Stylios, C.D., Groumpos, P.P.: An Integrated Two-Level Hierarchical Decision Making System based on Fuzzy Cognitive Maps (FCMs), IEEE Transactions on Biomedical Engineering, Vol. 50, No 12, pp. 1326-1339, December 2003.

6. Kosko, B.: Fuzzy Cognitive Maps, Int. J. Man-Machine Studies, Vol. 24 (1986), 65-75.

7. Aguilar, J.: Adaptive Random Fuzzy Cognitive Maps. In: Garijio, F.J., Riquelme, J.C., Toro, M. (eds.): IBERAMIA 2002, Lecture Notes in Artificial Intelligence 2527, SpringerVerlag Berlin Heidelberg (2002), 402-410.

8. Papageorgiou, E.I., Stylios, C.D., Groumpos, P.P.: Activation Hebbian Learning Rule for for Fuzzy Cognitive Map Learning, Proc. of $15^{\text {th }}$ IFAC World Congress of Inter. Federation of Automatic Control, Barcelona, Spain, July 21-26,CD-ROM, 2002.

9. Koulouriotis, D.E., Diakoulakis, I.E., Emiris, D.M.: Learning Fuzzy Cognitive Maps using evolution strategies: A novel schema for modeling a simulating high-level behavior. Proc. 2001 IEEE Congress on Evolutionary Compu., Seoul, Korea, 2001, vol. 1, pp. 364-371.

10. Oja, E., Ogawa, H., Wangviwattana, J.: Learning in nonlinear constrained Hebbian networks. In. T. Kohonen et al. (eds.): Artificial Neural Networks (1991), Amsterdam: North-Holland, 385-390. 